\title{
Comparison of Pedestrian Fundamental Diagram Across Cultures
}

\author{
Ujjal Chattaraj $^{1,2}$, Armin Seyfried ${ }^{1}$, Partha Chakroborty ${ }^{2}$ \\ ${ }^{1}$ Jülich Supercomputing Centre, Research Centre Jülich, 52425 Jülich, Germany \\ ${ }^{2}$ Indian Institute of Technology Kanpur, Kanpur 208 016, India \\ E-mail: ujjal@iitk.ac.in, a.seyfried@fz-juelich.de, partha@iitk.ac.in
}

March 1, 2009

\begin{abstract}
The relation between speed and density is connected with every self-organization phenomenon of pedestrian dynamics and offers the opportunity to analyze them quantitatively. But even for the simplest systems, like pedestrian streams in corridors, this fundamental relation isn't completely understood. Specifications of this characteristic in guidelines and text books differ considerably reflecting the contradictory database and the controversial discussion documented in the literature. In this contribution it is studied whether cultural influences and length of the corridor can be the causes for these deviations. To reduce as much as possible unintentioned effects, a system is chosen with reduced degrees of freedom and thus the most simple system, namely the movement of pedestrians along a line under closed boundary conditions. It is found that the speed of Indian test persons is less dependent on density than the speed of German test persons. Surprisingly the more unordered behaviour of the Indians is more effective than the ordered behaviour of the Germans. Without any statistical measure one cannot conclude about whether there are differences or not. By hypothesis test it is found quantitatively that these differences exist, suggesting cultural differences in the fundamental diagram of pedestrians.
\end{abstract}

Keywords: Pedestrian Traffic, Crowd Dynamics, Fundamental Diagram

\section{Introduction}

Dimension of pedestrian facilities is relevant in respect of comfort and safety in buildings with a large number of occupants (or people). Concerning comfort as well as safety several planning guidelines and text books are available $[1,2,3,4,4,6,6,7,8]$. In all these books or guidelines one basic characteristic to describe the dynamics of pedestrians is the relation between density and flow or density and speed, which following the terminology of vehicular traffic is also named the fundamental diagram. To illustrate the importance of the fundamental diagram two frequently asked questions that appear while designing of pedestrian facilities is discussed. In respect of safety it is important to know that how many pedestrians can pass a certain cross-section of the facility. Thus one wants to know how many pedestrians $\Delta N$ can pass the facility in a given time interval $\Delta t$. The quotient $\Delta N / \Delta t$ gives the flow $J$. Obviously the 
flow depends on the density. Another example is the calculation of travel times. Then one wants to know how long it will take to reach a certain destination, or an exit. The describing quantity is the speed which again will depend on the density. For low density one will be able to walk with the free flow speed. However with increasing density the speed will decrease and thus the travel-time increase. Thus the basic quantities to describe pedestrian stream are, the speed $v$, the density $\rho$ and the flow $J$. These quantities are related by the flow equation $J=\rho v w$ where $w$ gives the width of the facility and the empirical relation between flow and speed $J(v)$ or the relation between speed and density $v(\rho)$. For different facilities like stairs and corridors the shape of the diagrams differ, but in general it is assumed that the fundamental diagrams for the same type of facilities but different widths merge into one diagram for the specific flow $J_{s}=\frac{J}{w}$.
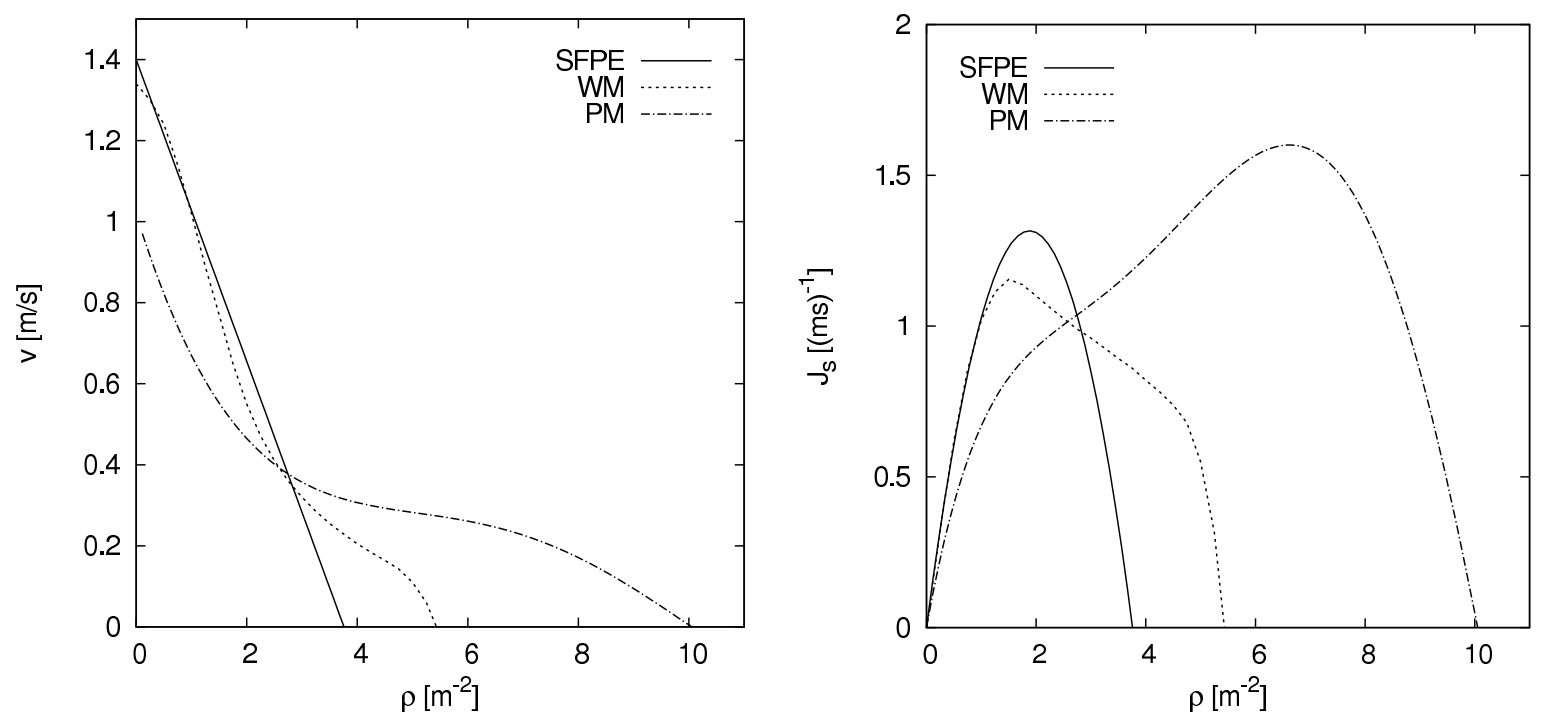

Figure 1: Specifications for the fundamental diagram in different guidelines. (SFPE: SFPE Handbook of Fire Protection Engineering [1, PM: Planning for foot traffic flow in buildings [6] and WM the guideline 'Transporttechnik der Fussgänger' [4].

Figure 1 shows various fundamental diagrams used in planing guidelines [6, 4, 5]. The comparison reveals that specifications disagree considerably. In particular the maximum of the function giving the capacity $J_{s, \max }$ ranges from $1.2(\mathrm{~ms})^{-1}$ to $1.8(\mathrm{~ms})^{-1}$, the density value where the maximum flow is reached $\rho_{c}$ (optimal density) ranges from $1.75 \mathrm{~m}^{-2}$ to $7 \mathrm{~m}^{-2}$ and, most notably, the density $\rho_{0}$ (jam density) where the speed approaches zero due to overcrowding ranges from $3.8 \mathrm{~m}^{-2}$ to $10 \mathrm{~m}^{-2}$. For a detailed discussion of the causes for these deviation refer to [9].

Several explanations for these deviations have been suggested, including cultural and population differences [10, 11, differences between uni- and multi-directional flow [12, 13, 14], short-ranged fluctuations [13], influence of psychological factors given by the incentive of the movement [6, 8] and, partially related to the latter, the type of traffic (commuters, shoppers) 2.

However there is no common agreement in the community which factors influence the relation and which do not. For example Weidmann [4] neglects differences between uni- and multidirectional flow in accordance with Fruin, who states in his often cited book [3] that the 
fundamental diagrams of multi-directional and unidirectional flow differ only slightly. This disagrees with results of Navin and Wheeler [12] and Lam et al. [14] who found a reduction of the flow due to directional imbalances. Unfortunately most of the authors do not give all informations and a comparison of different studies in general is subjected to uncontrollable errors and uncertainties. In addition it was shown in [15] that the measurement methods influence the resulting relations, restricting the number of comparable studies.

However, all diagrams agree in one characteristic: speed decreases with increasing density. So the discussion above indicates there are many possible reasons and causes for the speed reduction. For the movement of pedestrians along a line a linear relation between speed and the inverse of the density was measured in [16]. The speed for walking pedestrians depends also linearly on the length of a stride (or step size) [4] and the inverse of the density can be regarded as the required length of one pedestrian to move. Thus it seems that smaller step sizes caused by a reduction of the available space with increasing density is, at least for a certain density region, one cause for the decrease of speed.

The discussion above shows that there are many possible factors which influence the fundamental diagram. To identify the factors that influence the fundamental diagram it is necessary to exclude as much as possible, influences of measurement methodology and short range fluctuations on the data. Moreover the study presented in [16] showed that a reduction of the degrees of freedom gives an idea about the causes responsible for the speed reduction with density in pedestrian traffic.

In line with this approach experiments under laboratory conditions are performed and a system is chosen with smaller degrees of freedom to study whether and how cultural differences influences the speed density relation. The study of http://www.paceoflife.co.uk and Morral et. al. [10] is restricted to low densities $\rho<1 \mathrm{~m}^{-2}$ only. Thus up to now it is not sure whether cultural differences are present for the high density regime of the fundamental diagram. In addition whether length of corridor impacts the results or not is not studied yet. The problem at hand is to study pedestrian speed-distance headway data from Germany and India. Further, the intention is to analyze parameters such as free flow speed, minimum personal space and change of speed with distance headway, and see whether these vary between cultures. For this purpose experiments, as described in next section, were conducted in Germany and India. The data from these experiments are analyzed for the various parameters listed above and the analysis and results are presented later. The reason for studying speeddistance headway relationship instead of speed-density relationship is that it was intended to compare results from different cultures by some quantitative measure. This can only be done easily if the relationship is linear. It is a known fact and also was observed from the data speed-distance headway relationship can be reasonably described through a linear relation but speed-density can not be.

\section{Experimental Set-up and Data Collection}

\subsection{Experimental set-up}

The corridor is built up with chairs and ropes. The size and shape of the corridor is same as adopted in [16] for the similar experiment in Germany. The length of the corridor is $l_{p}=17.3$ $\mathrm{m}$ and that of the measured section is $l_{m}=2 \mathrm{~m}$. The measured section was constructed by erecting two ranging rods at the entry and exit line of the measured section, so that when a person is exactly at those positions the time can be noted. The camera was set at a sufficient 
distance of $10 \mathrm{~m}$ from the measured section along the perpendicular bisector of the measured section to reduce parallax error.

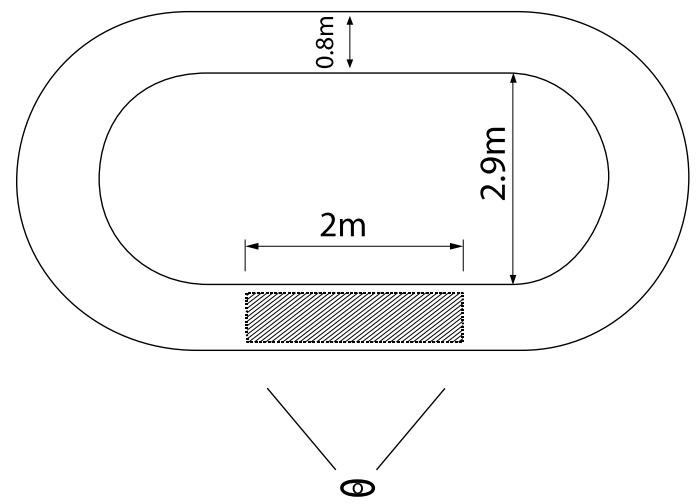

Figure 2: Sketch of the experimental set-up in India and Germany.

The channel in which pedestrian motion is studied is shown in Figure 2. Data however is collected only for the shaded section indicated in the figure. The width of the passageway in the straight section is $0.8 \mathrm{~m}$ which is sufficient for single file movement but not for overtaking. In the curved section the width is increased to a maximum of $1.2 \mathrm{~m}$ through elliptic transition curves. The reason for this is that the curved portion of the flow space may reduce speed. The experiment in India was done outdoor but on paved ground. The subjects for the experiment consisted of graduate students, technical staff of Indian Institute of Technology, Kanpur and local residents of Kanpur city thus putting variation in population. They were instructed not to overtake and not to push others. To obtain data on various densities six sets of experiments with number of subjects $N=1,15,20,25,30,34$ were performed. For the experiments (except $N=1$ ) all the subjects used in that cycle were initially distributed uniformly in the corridor. After the instruction to start was given every subject goes around the corridor two times. After that an opening is created in the closed corridor through which the subjects are allowed to leave and keep walking for a reasonable distance away from the corridor so that there is no tailback effect.
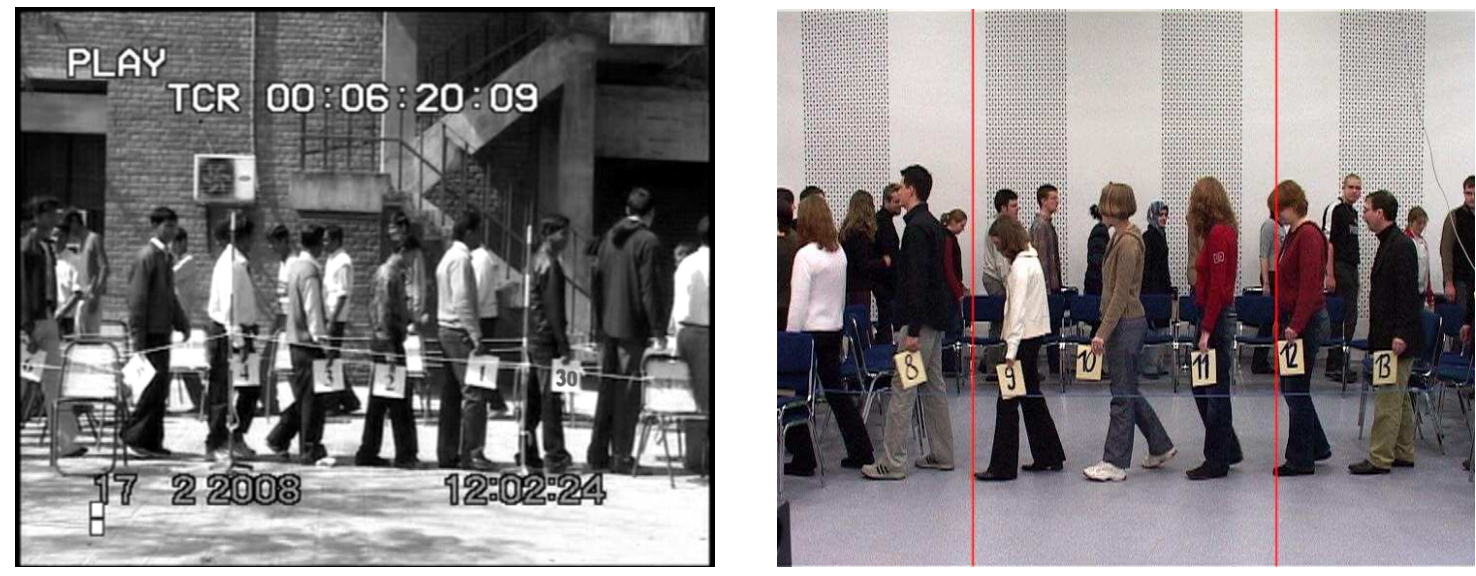

Figure 3: Snapshots for the run with $N=30$ Left: India Right: Germany.

The experimental set-up for the German study is described in detail in [16]. The length 
and the width of the corridor, the position and dimension of the measurement area, the number of subjects $N$ for the six sets, the instruction of the test persons, as well as the measurement method are identical to the study in India. Differences are the moving direction and the composition of the test subjects. In the German study the group of test persons was composed of male and female students and staff of the Institute. In the Indian study the group consist of male only. The moving direction of the experiment in India was clockwise while in Germany it was anti-clockwise. Figure 3 shows snapshots of the Indian and German experiment.

\subsection{Data collection}

Initially speed-density data were collected and later density was converted to distance headway by taking the reciprocal. To gather the speed-density data a digital video camera (25 frames/second) was stationed as shown in Figure 2. In the Indian experiment ranging rods were used to demarcate the measured section of Figure 2. A snapshot of the measured section obtained from video data is shown in Figure 3. From the video data times at which an individual (say individual $i$ ) entered $\left(t_{i}^{\text {in }}\right)$ and left $\left(t_{i}^{\text {out }}\right)$ this section are noted for every individual. The times are noted to the nearest 0.04 seconds. From this time points the information on speed and density are obtained. The German data were collected in the same way, only the ranging rods were not installed in the real set-up but lines added by image processing in the video, see Figure 3 .

\section{Analysis and Results}

\subsection{Time development}

Entrance and exit times $\left(t_{i}^{\text {in }}, t_{i}^{\text {out }}\right)$ were used to calculate the mean speed of individual persons $v_{i}=\frac{l_{m}}{t_{i}^{\text {out }}-t_{i}^{i n}}$ during the crossing of the measurement section and the density $\rho(t)=N(t) / l_{m}$, see Figure 4. The classical density shows strong fluctuation and thus the enhanced definition $\rho_{n}$ according to equation 1 in [16] is used. The developing of the density $\rho_{n}$ and the speed $v_{i}$ shows the distinct correlation of these quantities. For the Indian and the German experiment three phases can be identified: A starting phase, a steady state and an end phase. In the starting phase the pedestrian begin to walk and the value as well as the fluctuation of the speed differ from the stationary state. During the stationary state the fluctuations in the Indian data are higher than the fluctuations in the German data indicating a more unordered character of movement in the Indian data. The end phase, indicated by higher speeds starts once the corridor was opened and the persons leave the corridor. For the following analysis we restrict the data to the stationary state. The density assigned to the space mean speed of individual $i, v_{i}$ is the mean value of the density during the crossing $\rho_{i}=\int d t \rho_{n}(t)$. It is noted that the differences between the enhanced and the classical density in the mean value over time are negligible.

\subsection{Study on free flow speed}

The linear distance headway - speed $(h-v)$ plot of closed corridor can not predict free flow speed. However data on free flow speed were collected by making only one person move in the corridor. Mean free flow speed from Indian data and German data are $1.27 \mathrm{~ms}^{-1}$ (with 

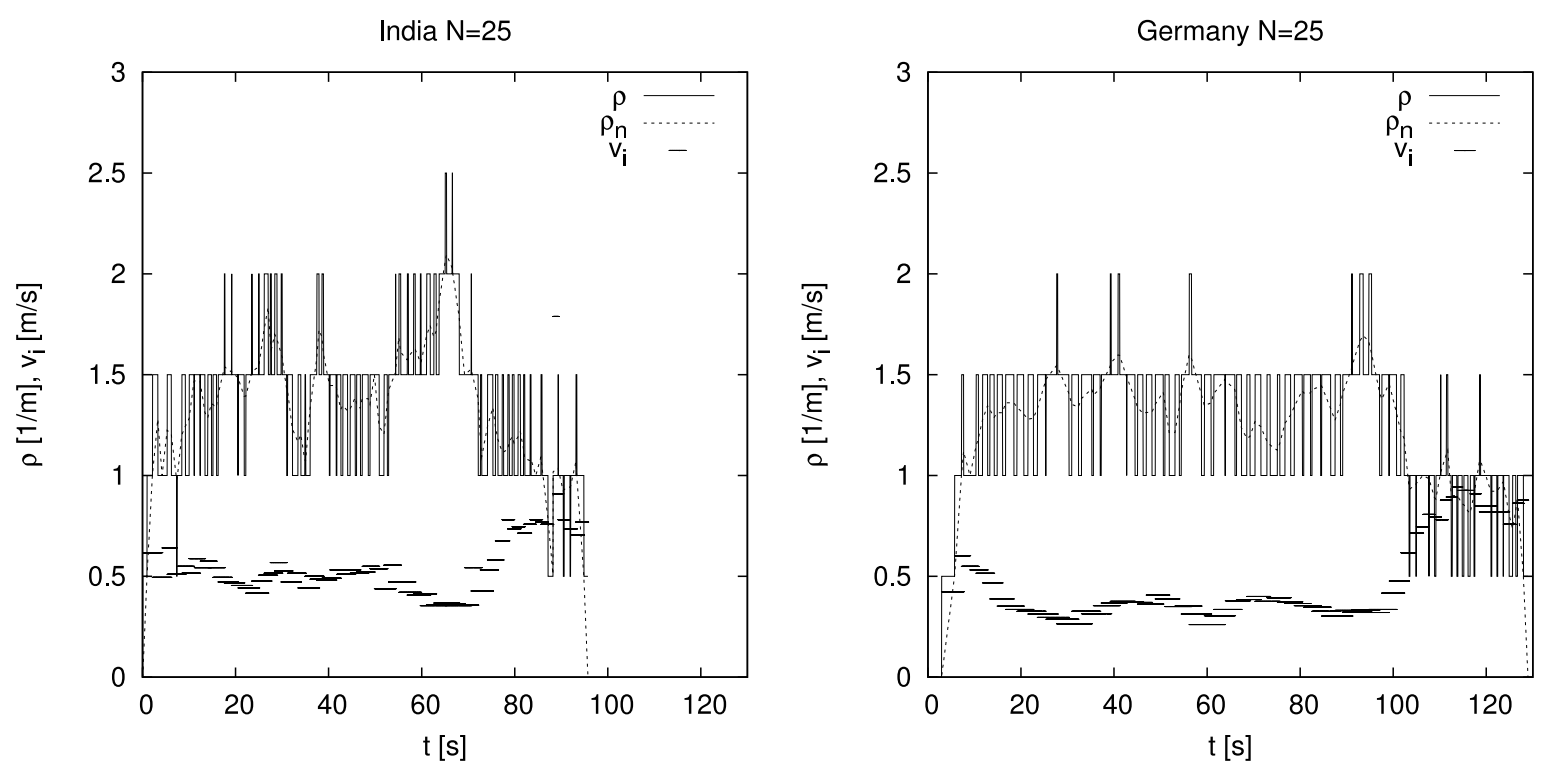

Figure 4: Time development of the speed $v_{i}$ and density $\rho$ for the sets with $N=25$. The length of the lines $v_{i}$ indicate the time interval the pedestrian is inside of the measurement area. The classical density $\rho$ shows strong fluctuations while $\rho_{n}$, the density according the enhanced definition introduced in [16], is smooth. For the Indian (left) and the German (right) cases three phases can be identified: A starting phase, a steady state and end phase where the subjects leave the corridor.

standard error of 0.03 ) and $1.24 \mathrm{~ms}^{-1}$ (with standard error of 0.02), respectively. Hypothesis testing on average free flow speed was conducted. Here lesser number of data points were obtained, so a $t$-test is appropriate for hypothesis testing, assuming normal distribution of data. The hypothesis that the mean free flow speed obtained from India $\left(v_{0}^{I}\right)$ and that from Germany $\left(v_{0}^{G}\right)$ are the same (i.e. Null hypothesis $H_{0}: v_{0}^{I}-v_{0}^{G}=0$ and alternate hypothesis $\left.H_{1}: v_{0}^{I}-v_{0}^{G} \neq 0\right)$ was tested. If the value of the expression

$$
t=\frac{v_{0}^{I}-v_{0}^{G}}{\sqrt{S_{v I}^{2}+S_{v G}^{2}}}
$$

comes out to be greater than some critical value at a certain level of confidence the Null hypothesis can be rejected saying that there is difference in free flow speed between Indian data and German data, otherwise not. Here, $S_{v I}$ and $S_{v G}$ are the standard errors for the free flow speed data from India and Germany, respectively. The value of the above expression for this data comes out to be 0.85 . The quantity obtained in equation 1 follows a $t$-distribution with $d f$ degrees of freedom. The value of $d f$ is given by Welch-Aspin equation as

$$
d f=\frac{\left(S_{v I}^{2}+S_{v G}^{2}\right)^{2}}{\frac{S_{v I}^{4}}{n_{I}-1}+\frac{S_{v G}^{4}}{n_{G}-1}}=33 .
$$

Where, $n_{I}$ and $n_{G}$ are the number of data points for Indian and German free flow speed data, respectively. For a two tailed $t$-test with a degree of freedom 33 the value of $t_{\text {critical }}$ is 2.03 at $95 \%$ level of confidence. Since the value of $t$ is less than $t_{\text {critical }}$ the Null hypothesis can not 
be rejected. The conclusion is that (mixed) population in Germany and (male) population in India have free flow speeds, which are not statistically different. This indicates that left to themselves both Germans and Indians walk in a similar manner.

\subsection{Speed - density relation across cultures}

The speed $(v)$ - density $(\rho)$ data in closed corridor condition obtained from India and Germany are plotted in Figure 5 .
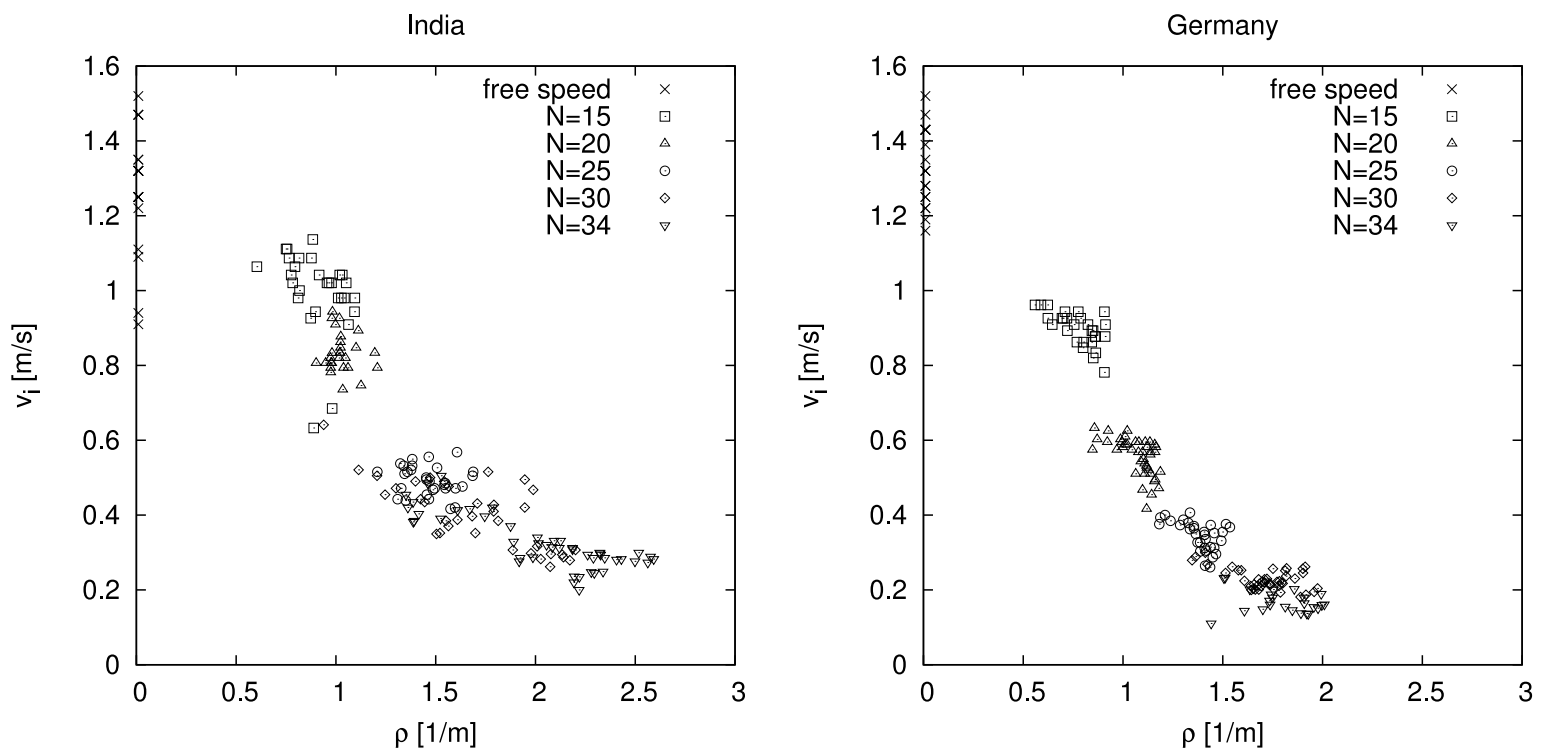

Figure 5: Speed-density data from Indian (left) and German (right) study, respectively.

On looking at the data the following subjective observations can be made. First, for every density region (except the free flow speed) Indians walk a bit faster than Germans. This will be discussed in detail in the next section. The free flow speed in India $v_{0}^{I}=1.27( \pm 0.16)$ $\mathrm{ms}^{-1}$ is same as the free flow speed in Germany $v_{0}^{G}=1.24( \pm 0.15) \mathrm{ms}^{-1}$. In spite of averaging over time the scatter of Indian data is larger than in Germany. e.g. for $N=15$ the speed ranges from $0.63 \mathrm{~ms}^{-1}$ to $1.11 \mathrm{~ms}^{-1}$ for India while in Germany the interval is restricted to $0.81 \mathrm{~ms}^{-1}$ to $0.96 \mathrm{~ms}^{-1}$. For larger $N$ this effect is much more pronounced; for example the density range for $N=34$ in India is $1.41 \mathrm{~m}^{-1}$ to $2.61 \mathrm{~m}^{-1}$ while in Germany it is $1.51 \mathrm{~m}^{-1}$ to $2.01 \mathrm{~m}^{-1}$. Thus the differences between individual crossing times and mean distances to adjacent persons for a fixed $N$ is higher in India indicating a more unordered character of the movement; even though the Indian composition of test persons is more homogeneous than the German group. This is affirmed by examination of the video recordings. Another difference is the rate of change of speed with change in density; this seems to be much higher for mid to high level densities (i.e., between 1 to $2 \mathrm{~m}^{-1}$ ) in Germany than in India.

But, the most important point observed here is that the speed-density relationship is not linear both for India and Germany, see Figure 5. On the other hand, the distance headway $(h)$ versus speed $(v)$ plot (Figure 6, right) is more suitable for description through a linear relationship. In order to study this observations made so far more closely first linear relationships are fitted to the Indian and German data on $h$ versus $v$ and their various hypotheses are tested on the estimated coefficients. 

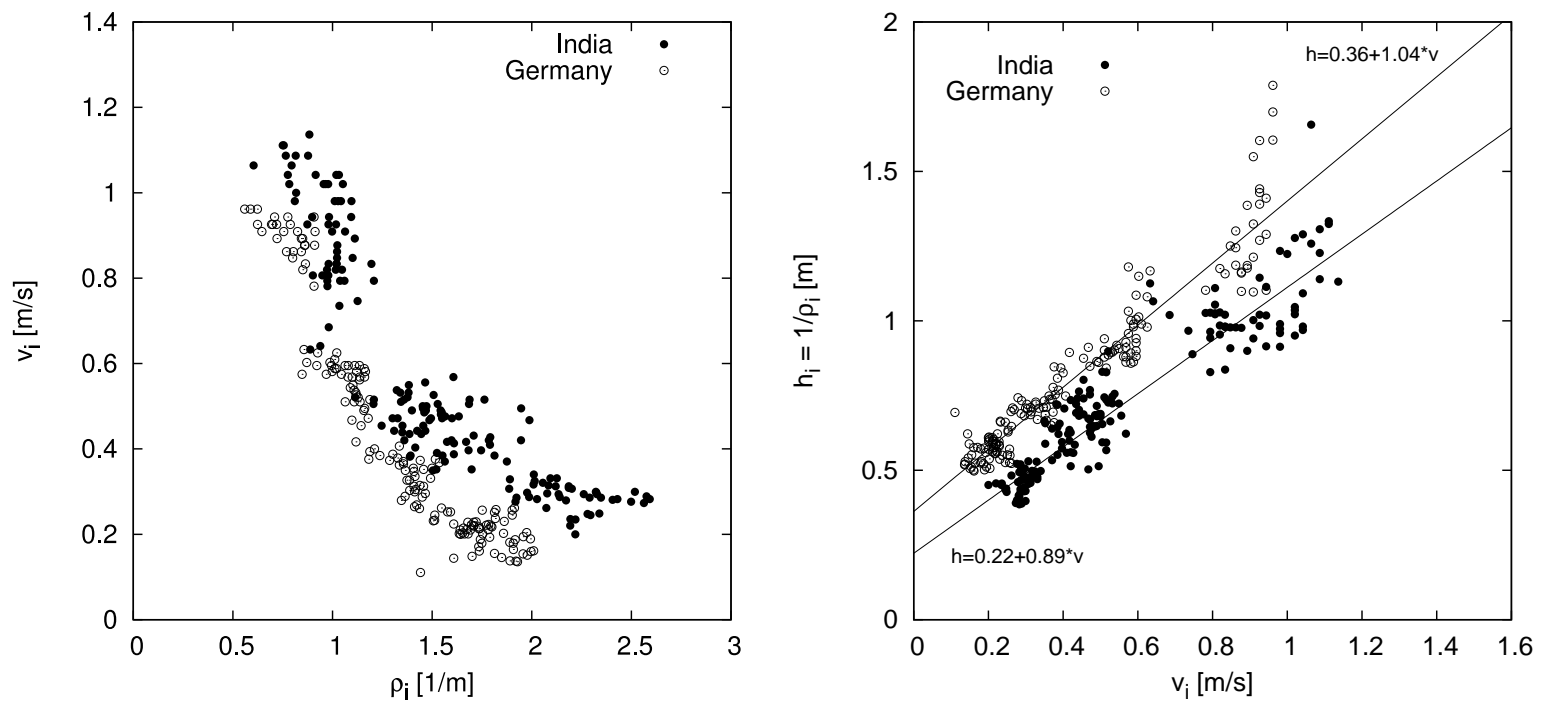

Figure 6: Left: Relation between speed $(v)$ and density $(\rho)$ from Indian and German study, respectively in closed condition. Right: shows the fitted linear $h-v$ relations for the Indian and German data, respectively. Table 1 presents more details of the regressed lines. As can be seen from the $R^{2}$ - values the simple linear relation $h=a+b v$ seems to explain much of the variation observed in the $h-v$ data.

\subsection{Speed-headway relation}

Relationships of the form $h=a+b v$ are fitted to the Indian and the German data. The values of $a$ and $b$ for the Indian and the German data are shown in Table1 1 along with other statistics. The fitted lines are also plotted with the data and are shown in Figure 6 (right). From the $t$-statistic values and the $R^{2}$ values shown in Table 1 it can be said that obtained fits are statistically sound. Note that in the relation $h=a+b v, a$ represents the minimum personal space and $b$ denotes the sensitivity of $h$ to $v$, or $\frac{d h}{d v}$.

\subsubsection{Study on minimum personal space}

As shown in Table 1 the estimated minimum personal space $a$ for India is $0.22 \mathrm{~m}$ and that for Germany is $0.36 \mathrm{~m}$. The standard errors of $a$ is $S_{a I}=0.02$ for India and $S_{a G}=0.01$ for Germany. The hypothesis that minimum personal space obtained from India, $a^{I}$ and that from Germany $a^{G}$ are the same (i.e., Null hypothesis $H_{0}: a^{G}-a^{I}=0$ and alternate hypothesis $H_{1}: a^{G}-a^{I} \neq 0$ ) was tested. Under the standard normality assumption and since the number of data points obtained from experiments was large the expression

$$
z=\frac{a^{G}-a^{I}}{\sqrt{S_{a G}^{2}+S_{a I}^{2}}}
$$

can be assumed to be normally distributed. $S_{a I}$ is the standard error for the data from India and $S_{a G}$ for Germany. If calculated $z$ value is more than the $z_{\text {critical }}$ value from table the Null hypothesis can be rejected, otherwise not.

For the above values of $a^{I}, a^{G}, S_{a I}$ and $S_{a G}, z$ from the above expression turns out to be 6.46. Since, this is more than 1.96, the $z_{\text {critical }}$ at $95 \%$ level of confidence for a two tailed 
test the Null hypothesis can be rejected. In other words estimated minimum personal space observed in India and Germany are statistically different. Here it is more for Germany than for India. Thus, one should observe higher jam density in India than in Germany.

\subsubsection{Study on change of distance headway with speed}

The coefficient $b$ represents the rate of change of distance headway $(h)$ with speed $(v)$. Alternatively this coefficient expresses how people react (in terms of speed) when the space ahead of them becomes restricted. As shown in the Table $1 b^{I}=0.89$ and $b^{G}=1.04$. The standard errors of $b$ is $S_{b I}=0.03$ for India and $S_{b G}=0.03$ for Germany. The Null hypothesis $\left(H_{0}\right.$ : $b^{G}-b^{I}=0$ and alternate hypothesis $\left.H_{1}: b^{G}-b^{I} \neq 0\right)$ was tested. The test proceeds along similar line, as expressed in the previous section. The $z$-value in this case comes out to be 3.99 and $z_{\text {critical }}$ at $95 \%$ level of confidence for a two tailed test is the same as in the previous case. Since $z>z_{\text {critical }}$, the Null hypothesis that there is no difference in $b$ for India and Germany can be rejected. Since $b^{G}$ is more than $b^{I}$, for increase in speed Germans require more space ahead than Indians. Thus one can say that Germans are more sensitive to high density conditions and decrease speed with increasing density more quicker than the Indians.

Table 1: Statistical measures for $h-v$ relationship in closed corridor over cultures.

\begin{tabular}{ccccc} 
Data set & $\begin{array}{c}\text { Intercept } \\
a[\mathrm{~m}]\end{array}$ & $\begin{array}{c}\text { Slope } \\
b[\mathrm{~s}]\end{array}$ & $\begin{array}{c}R^{2} \\
\text { \# data } \\
\text { points }\end{array}$ \\
\hline \hline India & 0.22 & 0.89 & 0.89 & 139 \\
$L_{\text {Cor }}=17.3 m$ & $(\mathrm{t}$ statistic $=12.76)$ & $(\mathrm{t}$ statistic $=32.99)$ & & \\
\hline Germany & 0.36 & 1.04 & 0.91 & 170 \\
$L_{\text {Cor }}=17.3 m$ & $(\mathrm{t}$ statistic $=28.02)$ & $(\mathrm{t}$ statistic $=40.33)$ & &
\end{tabular}

Table 2: Statistical measures for $h-v$ relationship in closed corridor over lengths of corridor.

\begin{tabular}{ccccc} 
Data set & $\begin{array}{c}\text { Intercept } \\
a[m]\end{array}$ & $\begin{array}{c}\text { Slope } \\
b[s]\end{array}$ & $\begin{array}{c}R^{2} \\
\text { \# data } \\
\text { points }\end{array}$ \\
\hline \hline India & 0.22 & 0.89 & 0.89 & 139 \\
$L_{C o r}=17.3 m$ & $($ t statistic $=12.76)$ & $($ t statistic $=32.99)$ & & \\
\hline India & 0.25 & 0.88 & 0.90 & 246 \\
$L_{\text {Cor }}=34.6 m$ & $($ t statistic $=22.83)$ & $($ t statistic $=47.53)$ & &
\end{tabular}

\subsection{Influence of corridor length}

The distance headway $(h)$-speed $(v)$ data in closed corridor for different length of corridor $l_{p}=17.3 \mathrm{~m}$ and $34.6 \mathrm{~m}$ obtained from India are plotted in Figure 7 . To achieve comparable densities for the double length of corridor we choose the following numbers of test persons: $N=30,40,50,60,68$. The values of $a, b$ and $R^{2}$ for a fitted relation $h=a+b v$ are as shown in Table 2, On conducting hypothesis tests on the difference of intercepts and on the difference 
of slope terms for the small length and large length section it was found that none of the differences are statistically significant. That is, length of the corridor has no impact on the $h-v$ relation.
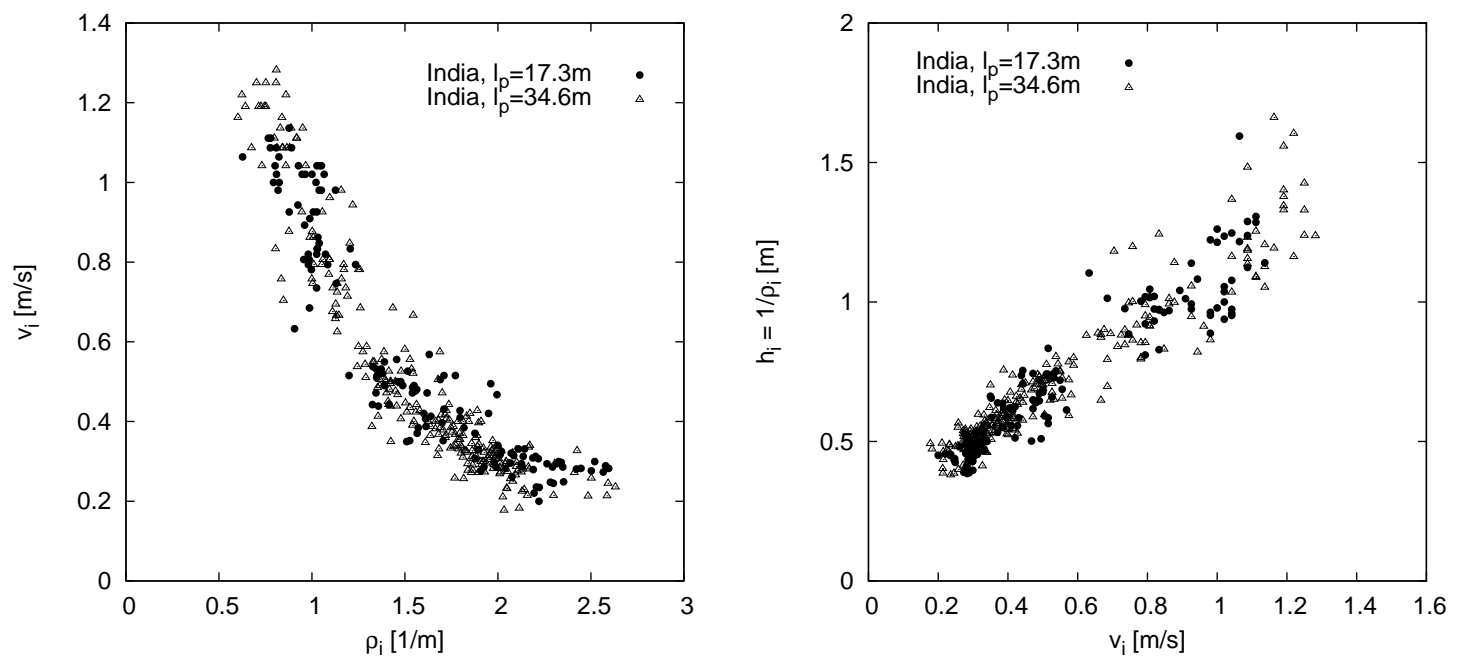

Figure 7: Speed-density data from India (Left) and speed-headway data from India (Right) for different lengths of corridor.

\section{Conclusions}

In this study fundamental diagrams from different cultures, namely German and Indian is presented. Fundamental diagrams for two different lengths of corridor is also presented. Same measurement method was followed throughout and results were compared statistically using $t$-test and $z$-test. The following are the observations: The free flow speed is the same for the Indian and the German group of test persons suggesting that when by themselves German and Indian pedestrians move in a similar manner. Estimated minimum personal space for the German group is more than that for Indian group indicating a higher jam density for India. Increase in distance headway with increase in speed for the German group is more than that of the Indian group indicating that Indians are less sensitive to increase in density compared to the Germans. It is believed that one cause for these differences is due to culture. For explanation of this statement the term security distance has to be introduced. The actual gap kept (i.e., the distance headway $h$ ) is composed of the required space for movement and the gap one wants to keep with his predecessor. This gap can be named as security distance. By visual inspection it appears that the Indian group of test persons are less concerned about the personal space of other persons and thus the security distance is smaller compared to the German group. To verify this it requires a method by which the dependence of fundamental diagram on security distance can be measured. Measurement of security distance from experimental data is difficult because the required space for movement changes temporarily due to the movement in steps. To test this idea it is planned to build a model where the security distance is a parameter. In addition for strong conclusion about cultural differences more experiments with various cultures and test group combinations are needed. Another important finding is that the length of the corridor does not have an effect on the fundamental 
diagram.

\section{Acknowledgment}

The authors are thankful to IIT Kanpur Transportation Laboratory (India) and Jülich Supercomputing Centre (Germany) for providing experimental facilities. The project was partially funded by the Deutsche Forschungsgemeinschaft (DFG) under Grant-Nr.: SE 1789/1-1.

\section{References}

[1] DiNenno PJ, SFPE Handbook of Fire Protection Engineering. National Fire Protection Association, Quincy MA, third edition, 2002.

[2] Oeding D, Verkehrsbelastung und Dimensionierung von Gehwegen und anderen Anlagen des Fußgängerverkehrs. Forschungsbericht 22, Technische Hochschule Braunschweig, 1963.

[3] Fruin JJ, Pedestrian Planning and Design. Elevator World, New York, 1971

[4] Weidmann U, Transporttechnik der Fussgänger. Technical Report Schriftenreihe des IVT Nr. 90, Institut für Verkehrsplanung, Transporttechnik, Strassen- und Eisenbahnbau, ETH Zürich, 1993. Zweite, ergänzte Auflage.

[5] Nelson HE, Mowrer FW, Emergency Movement. In P. J. DiNenno (Ed.), SFPE Handbook of Fire Protection Engineering, Chapter 14:367-380. National Fire Protection Association, Quincy MA, Third Edition, 2002.

[6] Predtechenskii VM, Milinskii AI, Planning for Foot Traffic Flow in Buildings. Amerind Publishing, New Dehli, 1978. Translation of: Proekttirovanie Zhdanii s Uchetom Organizatsii Dvizheniya Lyuddskikh Potokov, Stroiizdat Publishers, Moscow, 1969.

[7] Highway Capacity Manual. Technical report, Transportation Research Board, Washington DC, 1985.

[8] Kholshevnikov VV, Shields TJ, Boyce KE, Samoshin DA, Recent Developments in Pedestrian Flow Theory and Research in Russia. Fire Safety Journal 2008, 43(2):108-118.

[9] Schadschneider A, Klingsch W, Kluepfel H, Kretz T, Rogsch C, Seyfried A, Evacuation Dynamics: Empirical Results, Modeling and Applications: In Meyers, R. (Ed.) Encyclopedia of Complexity and System Science, Springer New York, to appear 2009.

[10] Morrall JF, Ratnayake LL, Seneviratne PN, Comparison of CBD Pedestrian Characteristics in Canada and Sri Lanka. Transportation Research Record 1991, 1294:57-61.

[11] Helbing D, Johansson A, Al-Abideen HZ, Dynamics of Crowd Disasters: An Empirical Study. Physical Review E 2007; 75:046109.

[12] Navin FD, Wheeler RJ, Pedestrian Flow Characteristics. Traffic Engineering 1969, $39: 31-36$. 
[13] Pushkarev B, Zupan JM, Capacity of Walkways. Transportation Research Record 1975, $538: 1-15$.

[14] Lam WHK, Lee JYS, Chan KS, Goh PK, A Generalised Function for Modeling Bidirectional Flow Effects on Indoor Walkways in Hong Kong. Transportation Research Part A: Policy and Practice 2003, 37:789-810.

[15] Seyfried A, Boltes M, Kähler J, Klingsch W, Portz A, Schadschneider A, Steffen B, Winkens A, Enhanced Empirical Data for the Fundamental Diagram and the Flow through Bottlenecks. In Pedestrian and Evacuation Dynamics 2008. Springer, 2008.

[16] Seyfried A, Steffen B, Klingsch W, Boltes M, The Fundamental Diagram of Pedestrian Movement Revisited. J. Stat. Mech. 2005, P10002. 